Micro-reflectance and transmittance spectroscopy: a versatile and powerful tool to characterize 2D materials

This content has been downloaded from IOPscience. Please scroll down to see the full text.

2017 J. Phys. D: Appl. Phys. 50074002

(http://iopscience.iop.org/0022-3727/50/7/074002)

View the table of contents for this issue, or go to the journal homepage for more

Download details:

IP Address: 128.179.136.176

This content was downloaded on 27/03/2017 at 15:11

Please note that terms and conditions apply.

You may also be interested in:

A direct comparison of CVD-grown and exfoliated MoS2 using optical spectroscopy

G Plechinger, J Mann, E Preciado et al.

Reversible uniaxial strain tuning in atomically thin WSe2

Robert Schmidt, Iris Niehues, Robert Schneider et al.

Light-matter interaction in transition metal dichalcogenides and their heterostructures

Ursula Wurstbauer, Bastian Miller, Eric Parzinger et al.

Isolation and characterization of few-layer black phosphorus

Andres Castellanos-Gomez, Leonardo Vicarelli, Elsa Prada et al.

Influence of the substrate material on the optical properties of tungsten diselenide monolayers

Sina Lippert, Lorenz Maximilian Schneider, Dylan Renaud et al.

Optical spectroscopy of interlayer coupling in artificially stacked MoS2 layers

G Plechinger, F Mooshammer, A Castellanos-Gomez et al.

Review of ultrafast spectroscopy studies of valley carrier dynamics in two-dimensional semiconducting transition metal dichalcogenides

Dong Sun, Jia-Wei Lai, Jun-Chao Ma et al.

Raman spectroscopy of transition metal dichalcogenides

$\mathrm{R}$ Saito, $\mathrm{Y}$ Tatsumi, S Huang et al.

Strain engineering in semiconducting two-dimensional crystals

Rafael Roldán, Andrés Castellanos-Gomez, Emmanuele Cappelluti et al. 


\title{
Micro-reflectance and transmittance spectroscopy: a versatile and powerful tool to characterize 2D materials
}

\author{
Riccardo Frisenda ${ }^{1}$, Yue Niu ${ }^{1,2}$, Patricia Gant ${ }^{1}$, Aday J Molina-Mendoza ${ }^{1}$, \\ Robert Schmidt ${ }^{3}$, Rudolf Bratschitsch ${ }^{3}$, Jinxin Liu ${ }^{4}$, Lei Fu ${ }^{4}$, \\ Dumitru Dumcenco ${ }^{5,6}$, Andras Kis ${ }^{5,6}$, David Perez De Lara ${ }^{1}$ \\ and Andres Castellanos-Gomez ${ }^{1}$
}

\footnotetext{
${ }^{1}$ Instituto Madrileño de Estudios Avanzados en Nanociencia (IMDEA Nanociencia), Campus de Cantoblanco, E-28049 Madrid, Spain

2 National Key Laboratory of Science and Technology on Advanced Composites in Special Environments, Harbin Institute of Technology, Harbin, People's Republic of China

${ }^{3}$ Institute of Physics and Center for Nanotechnology, University of Münster, 48149 Münster, Germany

${ }^{4}$ Laboratory of Advanced Nanomaterials, College of Chemistry and Molecular Science. Wuhan University, Wuhan, People's Republic of China

${ }^{5}$ Electrical Engineering Institute, École Polytechnique Fédérale de Lausanne (EPFL), CH-1015

Lausanne, Switzerland

${ }^{6}$ Institute of Materials Science and Engineering, École Polytechnique Fédérale de Lausanne (EPFL),

CH-1015 Lausanne, Switzerland

E-mail: andres.castellanos@imdea.org
}

Received 10 October 2016, revised 28 November 2016

Accepted for publication 7 December 2016

Published 23 January 2017

\begin{abstract}
Optical spectroscopy techniques such as differential reflectance and transmittance have proven to be very powerful techniques for studying 2D materials. However, a thorough description of the experimental setups needed to carry out these measurements is lacking in the literature. We describe a versatile optical microscope setup for carrying out differential reflectance and transmittance spectroscopy in 2D materials with a lateral resolution of $\sim 1 \mu \mathrm{m}$ in the visible and near-infrared part of the spectrum. We demonstrate the potential of the presented setup to determine the number of layers of 2D materials and characterize their fundamental optical properties, such as excitonic resonances. We illustrate its performance by studying mechanically exfoliated and chemical vapor-deposited transition metal dichalcogenide samples.
\end{abstract}

Keywords: 2D materials, optical spectroscopy, differential reflectance, transmittance, transition metal dichalcogenides

\section{S. Supplementary material for this article is available online}

(Some figures may appear in colour only in the online journal)

The use of optical microscopy-based characterization techniques is closely bound up with the birth and growth of the field of 2D materials [1-5]. These techniques are widely used since they are fast and simple to implement and, very importantly, they are non-destructive. Quantitative studies of the optical contrast of flakes deposited on $\mathrm{SiO}_{2} / \mathrm{Si}$ substrates were used first to provide a coarse estimate of the thickness of mechanically exfoliated flakes [3, 6-8]. Raman [2,9] and photoluminescence spectroscopy $[4,5,10-13]$ have proven to be very powerful techniques to determine the number of layers of 2D materials and study their intrinsic optical properties. 

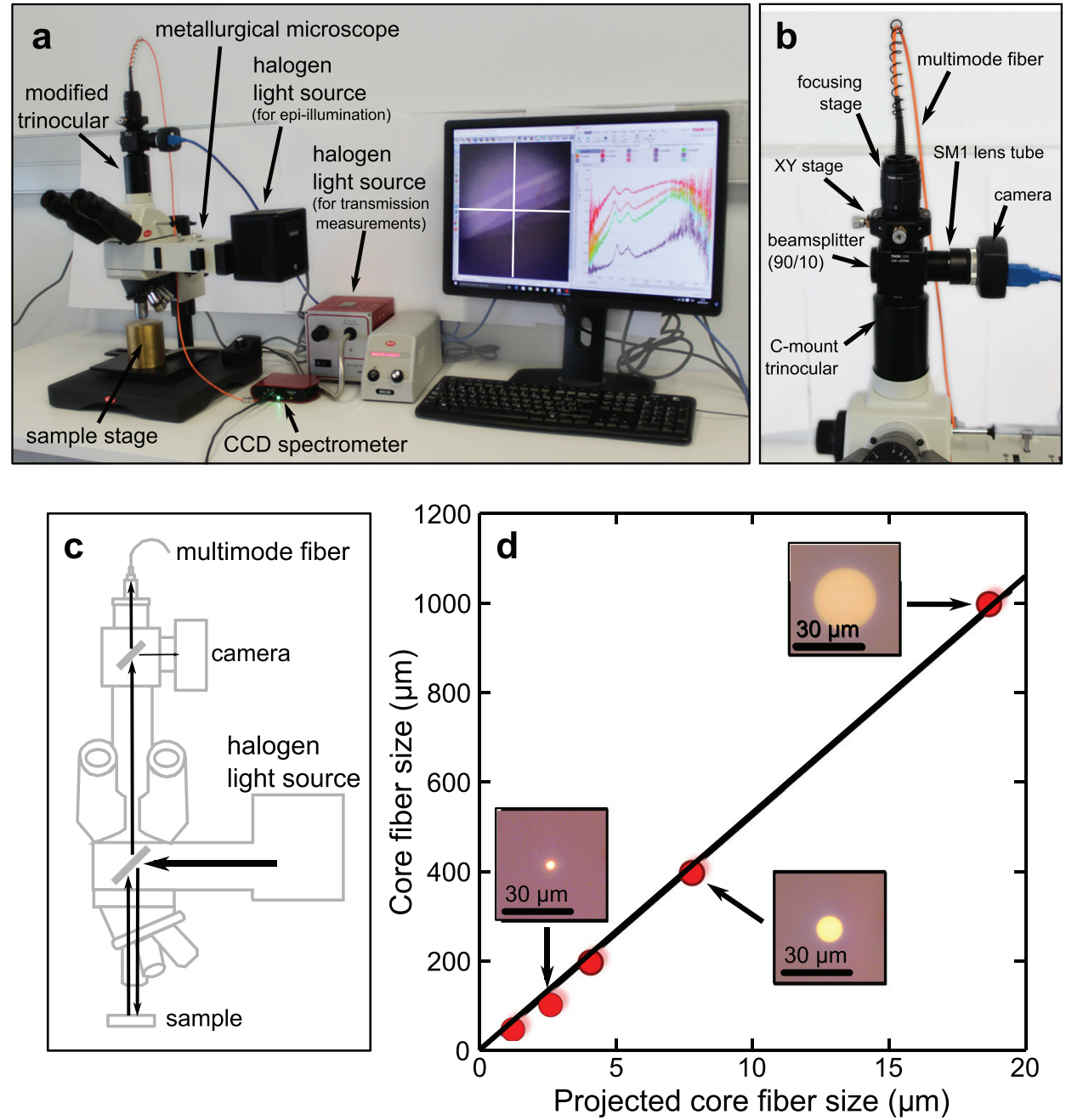

Figure 1. (a) Photograph of the experimental setup for performing micro-reflectance and transmittance spectroscopy on 2D materials, highlighting some of the key components. (b) Zoomed photograph of the modified trinocular. (c) Schematic diagram of the optical path of the setup operated in reflection mode. (d) Relationship between the size of the fiber core and its image on the sample surface due to the magnification of the optical system.

Differential reflectance has also been used recently to study the optical properties of 2D materials, especially transition metal dichalcogenides (TMDCs) [12, 14-17]. This technique provides a powerful tool to characterize $2 \mathrm{D}$ materials (even those that do not present strong excitonic features or Raman modes) in a broad range of the electromagnetic spectrum. Nevertheless, a thorough description of optical setups for characterizing 2D materials by differential reflectance is still lacking in the literature. This is the aim of this work: to accurately describe a versatile optical microscopy setup developed to carry out differential reflectance and transmittance measurements on $2 \mathrm{D}$ materials with $\sim 1 \mu \mathrm{m}$ spatial resolution in the wavelength range of $400 \mathrm{~nm}-900 \mathrm{~nm}(1.4 \mathrm{eV}-3.1 \mathrm{eV})$. The entire system can be replicated easily with a relatively low investment $(<9000 €)$. Moreover, existing optical microscopy setups could easily be upgraded to carry out these spectroscopic measurements.

\section{Components of the experimental setup}

Figure 1(a) shows a photograph of the micro-reflectance/ transmittance setup that we developed to characterize 2D materials. The setup consists of a Motic BA310 metallurgical microscope, a modified trinocular port, a halogen light source with a liquid light guide and a fiber-coupled CCD spectrometer. Figure 1(b) shows a detailed photograph of the modified trinocular, indicating the different components employed for its assembly. A 90:10 beam splitter is connected to the $\mathrm{C}$-mount trinocular in order to divide the trinocular light beam into two paths. In one of the paths $(10 \%$ of the intensity) a CMOS camera is placed at the image plane of the optical system to acquire images of the studied sample. In the other path ( $90 \%$ of the intensity) we place a multimode fiber, also at the image plane, projecting an image of the studied sample onto the surface of the fiber end. Table 1 summarizes the complete list of components necessary to assemble the experimental setup, including their part number, distributor and purchase price.

\section{Setting up the microscope}

The size of the fiber core acts as a confocal pinhole, and thus the fiber only collects the light coming from a small region of the sample. In order to determine the area and position of this region one can connect the free end of the multimode fiber to 
Table 1. List of the components employed to assemble the experimental setup.

\begin{tabular}{lllr}
\hline Part number & Description & Distributor & Price $(€)$ \\
\hline BA310Met-H Trinocular & Metallurgical microscope (with epi-illumination) & Motic & 3620.00 \\
CCS200/M & CCD spectrometer & Thorlabs & 2475.00 \\
OSL2 & Halogen lamp with fiber bundle & Thorlabs & 807.30 \\
SM1SMA & Adaptor to connect the multimode fiber to the halogen lamp & Thorlabs & 25.22 \\
Adaptor BA series & C-mount threaded trinocular & Motic & 127.00 \\
SM1A10 & Adaptor to connect the beam splitter mount to the C-mount trinocular & Thorlabs & 16.88 \\
CCM1-4ER/M & Mount for beam splitter cube & Thorlabs & 115.20 \\
BS025 & $90: 10$ beam splitter cube & Thorlabs & 186.30 \\
SM1A10 & Adaptor to connect the beam splitter mount to the XY stage & Thorlabs & 16.88 \\
CXY1 & XY stage & Thorlabs & 152.15 \\
SM1NR05 & Focusing stage & Thorlabs & 162.00 \\
SM05SMA & Adaptor to connect the multimode fiber to the focusing stage & Thorlabs & 24.57 \\
M96L01 & 105 $\mu$ m core multimode fiber & Thorlabs & 95.53 \\
SM1V10 & SM1 lens tube to connect to the beam splitter mount & Thorlabs & 29.34 \\
SM1A39 & Adaptor to connect the SM1 lens tube to the CMOS camera & Thorlabs & 18.00 \\
$\#$ 89-734 & CMOS camera & Edmund optics & 563.50 \\
- & Homemade brass sample holder with a hole for the fiber bundle & Workshop & 150.00 \\
& & Total & 8584.87 \\
\hline
\end{tabular}
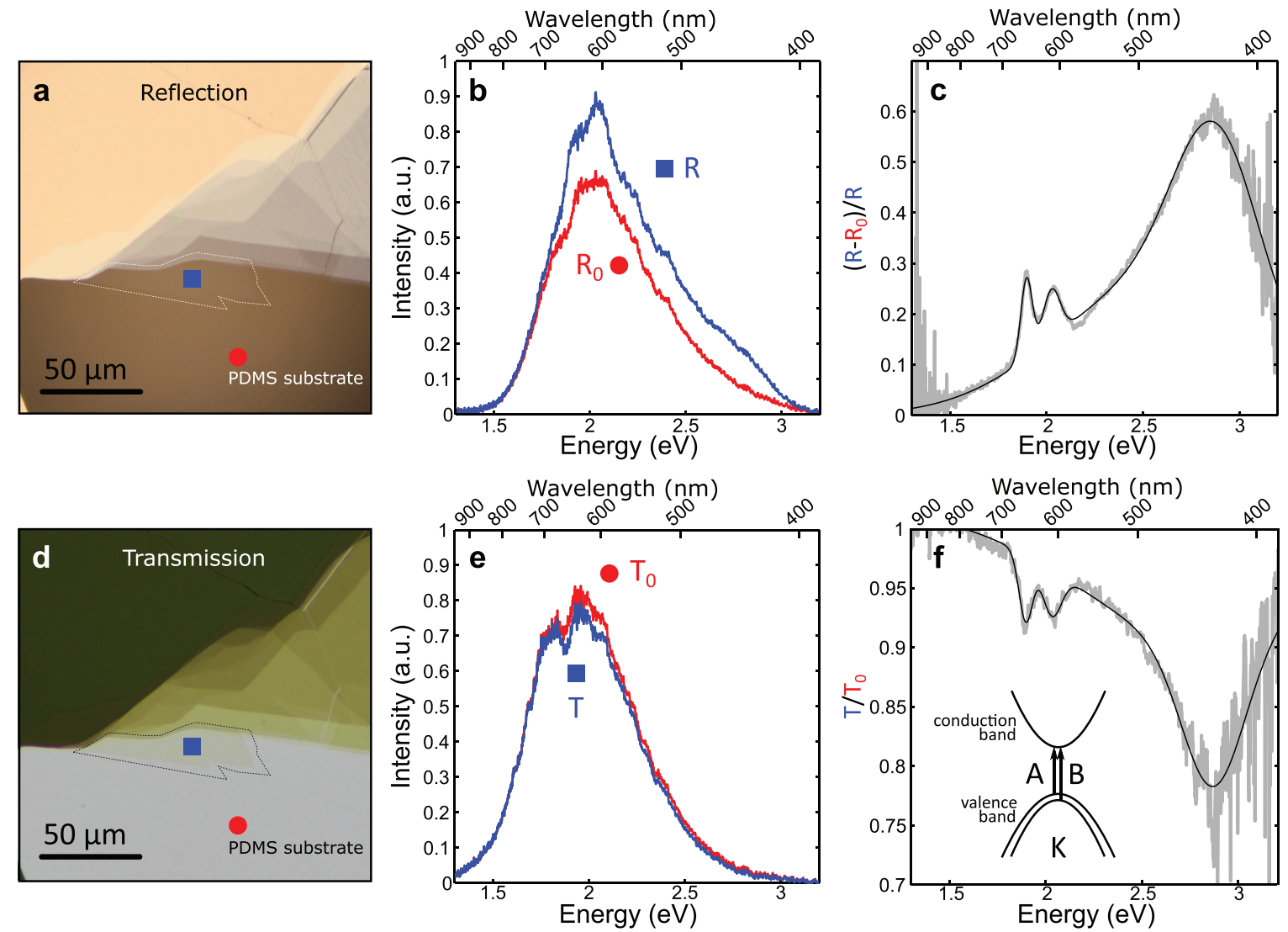

Figure 2. (a) Optical reflection image of an $\mathrm{MoS}_{2}$ flake transferred onto a PDMS substrate (epi-illumination). The single layer area is highlighted with a dashed line. (b) Reflection spectra acquired at the positions marked with the blue square and red circle in (a). (c) Calculated differential reflectance of the single layer $\mathrm{MoS}_{2}$ from the spectra displayed in (b). The thin black line is a multi-Gaussian fit used to extract the peak position and full width at half maximum values of the different excitons. (d) Transmission mode optical image of the flake shown in (a). (e) Transmission spectra acquired on the flake (blue square) and substrate (red circle) are employed to calculate the transmittance spectrum of the single layer flake (f). The inset in (f) shows a simplified schematic of the band diagram around the $\mathrm{K}$ point that illustrates the origin of the two excitonic resonances at $1.90 \mathrm{eV}$ and $2.05 \mathrm{eV}$. 

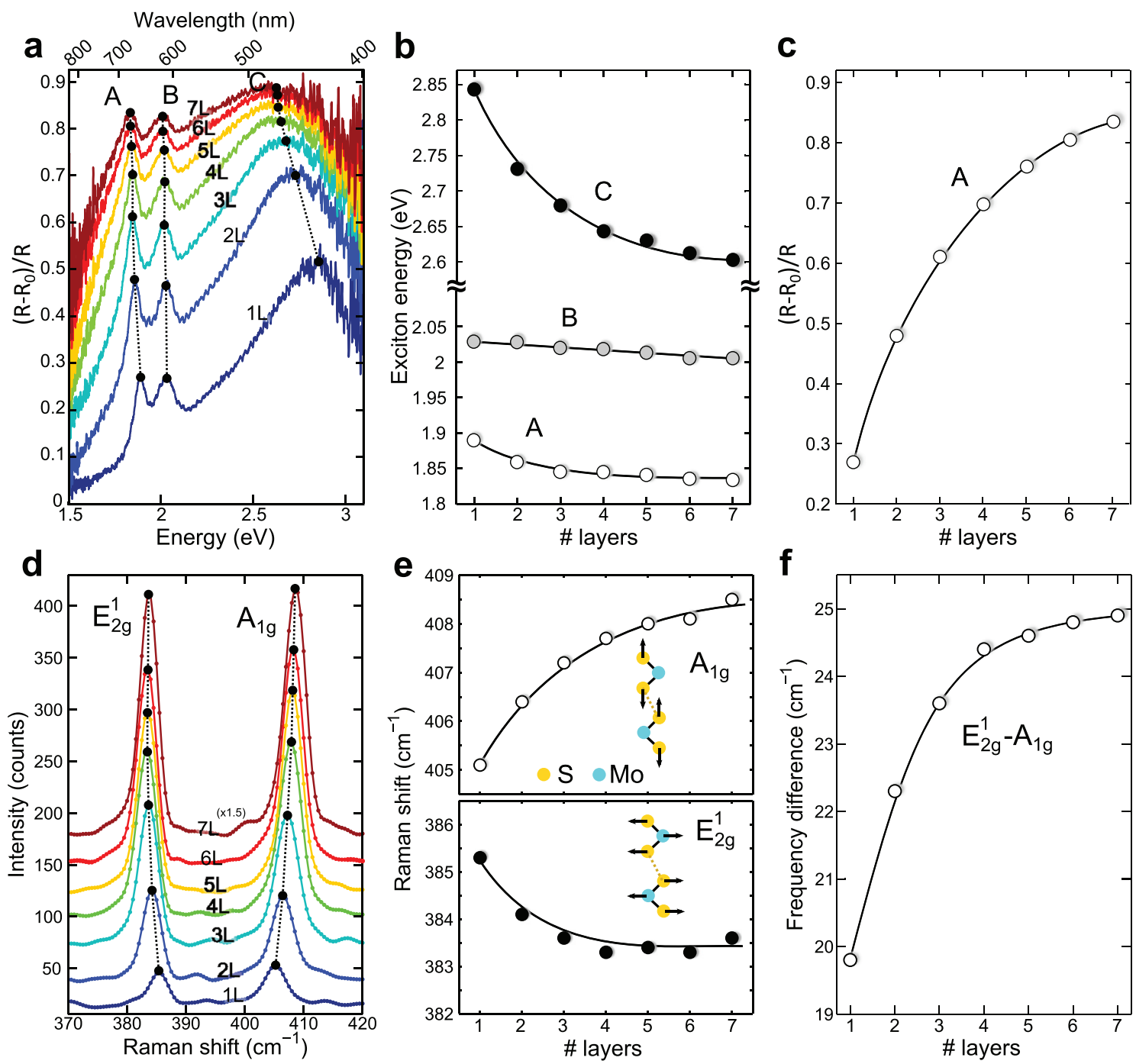

Figure 3. (a) Differential reflectance spectra acquired for $\mathrm{MoS}_{2}$ flakes with different numbers of layers. The peak positions of the different excitons are highlighted by filled circles. (b) Thickness dependence of the exciton energy. (c) Amplitude of the A exciton peak as a function of the number of layers. (d) Raman spectra acquired for $\mathrm{MoS}_{2}$ flakes with different numbers of layers. The peak positions of the two most prominent Raman modes are highlighted by filled circles. (e) Thickness dependence of the Raman shift of the $A_{1 \mathrm{~g}}$ and $E_{2 \mathrm{~g}}^{1}$ modes. The insets in (d) show an artistic representation of the vibrational modes. (f) Frequency difference between the Raman modes as a function of the number of layers.

a white light source (e.g. the halogen light source), yielding an image of the fiber end facet in the sample plane. The image of the fiber core at the end facet is projected according to the magnification of the microscope lenses and objective assembly. Figure 1(d) shows the relationship between the core diameter of the different multimode fibers and their apparent size in the sample plane. An $X Y$ stage allows one to align the image of the end facet with the digital crosshair of the camera that marks the center of the sample image. With a reversed beam path (see figure 1(c)), this corresponds to the light collection spot and it is possible to determine where the spectra are collected once the light source is disconnected from the fiber end. A focusing stage in the trinocular also allows one to accurately place the fiber core end right in the image plane by moving the focusing stage until the projected fiber core image and the surface of the studied sample are both perfectly in focus. Once the size for light collection has been determined, centered in the crosshair and focused, the fiber is disconnected from the white light source and the free end of the fiber is connected to the fiber-coupled CCD spectrometer.

\section{Acquisition of micro-reflectance or transmittance spectra}

To acquire a micro-reflectance spectrum the microscope is operated in epi-illumination mode. The white light of the microscope halogen lamp is reflected onto the sample, collected through a long working distance (infinity corrected) $50 \times$ microscope objective (NA 0.55) and directed to the modified trinocular. In epi-illumination operation, closing the field aperture of the microscope (to $\sim 60 \mu \mathrm{m}$ in diameter) reduces the stray light collected by the finite numerical aperture of the objective lens. Transmittance spectra can also be acquired by turning off the microscope lamp and placing the sample onto a sample holder connected to the halogen light source by a fiber bundle (NA 0.57). The sample holder has a see-through 
hole where the fiber bundle can be slid in. The sample is directly placed onto the end facet of the fiber bundle without using other lenses (see the supplementary material, available at stacks.iop.org/JPhysD/50/074002/mmedia).

\section{Experimental results for monolayer and few-layer TMDCs}

Figure 2(a) shows a reflection mode optical image of a $\mathrm{MoS}_{2}$ flake deposited onto a PDMS substrate (gel film from Gel-Pak ${ }^{\circledR}$ ) by mechanical exfoliation of bulk $\mathrm{MoS}_{2}$ (Moly Hill mine, Quebec, QC, Canada) with Nitto tape (Nitto Denko Co., SPV224 clear). The single-layer region of the flake is highlighted with a dashed line to facilitate its identification. Figure 2(b) shows the spectra acquired at the positions marked in the optical image of figure 2(a) with a blue square (flake, $R$ ) and a red circle (substrate, $R_{0}$ ). The differential reflectance spectrum is then calculated as $\left(R-R_{0}\right) / R$ (see figure $2(\mathrm{c})$ ) and it is related to the absorption coefficient of the material $\alpha(\lambda)$ as $[14,18]$

$$
\frac{R-R_{0}}{R}=\frac{4 n}{n_{0}^{2}-1} \alpha(\lambda),
$$

where $n$ is the refractive index of the flake under study and $n_{0}$ is the refractive index of the substrate. Figure 2(d) shows a transmission mode optical image of the flake shown in figure 2(a). Figure 2(e) shows the transmission spectra acquired at the positions highlighted with a blue square (flake, $T$ ) and red circle (substrate, $T_{0}$ ) in figure $2(\mathrm{~d})$. The flake transmittance can be determined directly by dividing $T / T_{0}$ (see figure $2(\mathrm{f})$ ).

Both the differential reflectance and transmittance spectra have two prominent narrow peaks/dips occurring at photon energies $\sim 1.90 \mathrm{eV}$ and $\sim 2.05 \mathrm{eV}$, which correspond to the optical absorption due to the direct transitions at the $\mathrm{K}$ point of the Brillouin zone and are associated with the generation of the A and B excitons, respectively. This observation is in agreement with previous photoluminescence studies [4, 5, 19]. The inset in figure 2(f) shows a simplified band diagram at the $\mathrm{K}$ point of the Brillouin zone, where the origin of the $\mathrm{A}$ and $\mathrm{B}$ excitonic resonances is illustrated. The valence band is split because the strong spin-orbit interaction due to the Mo atoms gives rise to two direct band gap transitions at the $\mathrm{K}$ point. The spectra also show a broad feature around $2.85 \mathrm{eV}$. This feature is not typically observed in photoluminescence experiments, which mostly use an excitation wavelength of $\sim 2.3 \mathrm{eV}$. Recent hyperspectral, reflectance, ellipsometry and photocurrent spectroscopy experiments, however, present this feature (referred to as the $\mathrm{C}$ exciton peak). It originates from singularities in the joint density of states between the first valence and conduction bands near the $\Gamma$ point of the valence band, which lead to multiple optical transitions that are nearly degenerate in energy [14, 15, 20-24].

Micro-reflectance and transmittance are interesting techniques for determining the number of layers of 2D materials. Figure 3(a) shows, as an example, the differential reflectance spectra acquired for $\mathrm{MoS}_{2}$ flakes with a thickness ranging from one to seven layers. One can determine the number of

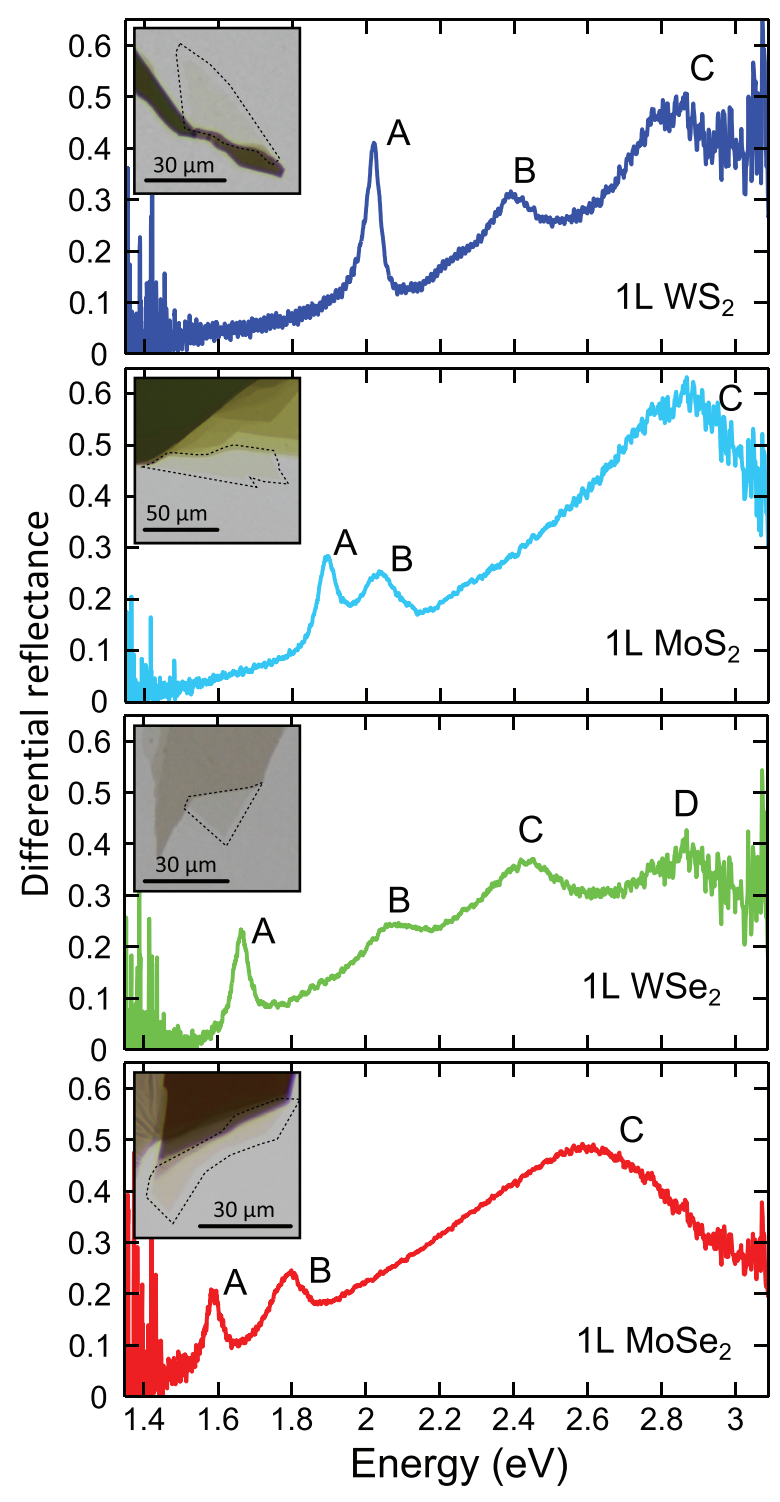

Figure 4. Differential reflectance spectra acquired for single-layer flakes of different transition metal dichalcogenides: $\mathrm{WS}_{2}, \mathrm{MoS}_{2}$, $\mathrm{WSe}_{2}$ and $\mathrm{MoSe}_{2}$ (from top to bottom). The exciton resonances are highlighted according to the notation employed in the literature $[15,27]$. (Insets) Transmission mode optical images of the studied single-layer flakes (highlighted with a dashed line).

Table 2. Summary of the A and B exciton energies extracted from the differential reflectance measurements shown in figure 4 .

\begin{tabular}{|c|c|c|}
\hline Material & A exciton $(\mathrm{eV})$ & B exciton $(\mathrm{eV})$ \\
\hline $1 \mathrm{~L} \mathrm{WS}{ }_{2}$ & 2.02 & 2.39 \\
\hline $1 \mathrm{~L} \mathrm{MoS}_{2}$ & 1.90 & 2.04 \\
\hline $1 \mathrm{~L} \mathrm{WSe}_{2}$ & 1.66 & 2.06 \\
\hline $1 \mathrm{~L} \mathrm{MoSe}_{2}$ & 1.59 & 1.80 \\
\hline
\end{tabular}

layers from the energy of the A exciton, which blue-shifts upon reduction of the number of layers (see figure 3(b)) [25]. The B exciton, on the other hand, shows a weaker thickness dependence [25]. These results are in good agreement with previous photoluminescence measurements [5]. Interestingly, the $\mathrm{C}$ exciton peak also shifts with the number of layers and its dependence is even stronger than that of the A exciton; one 

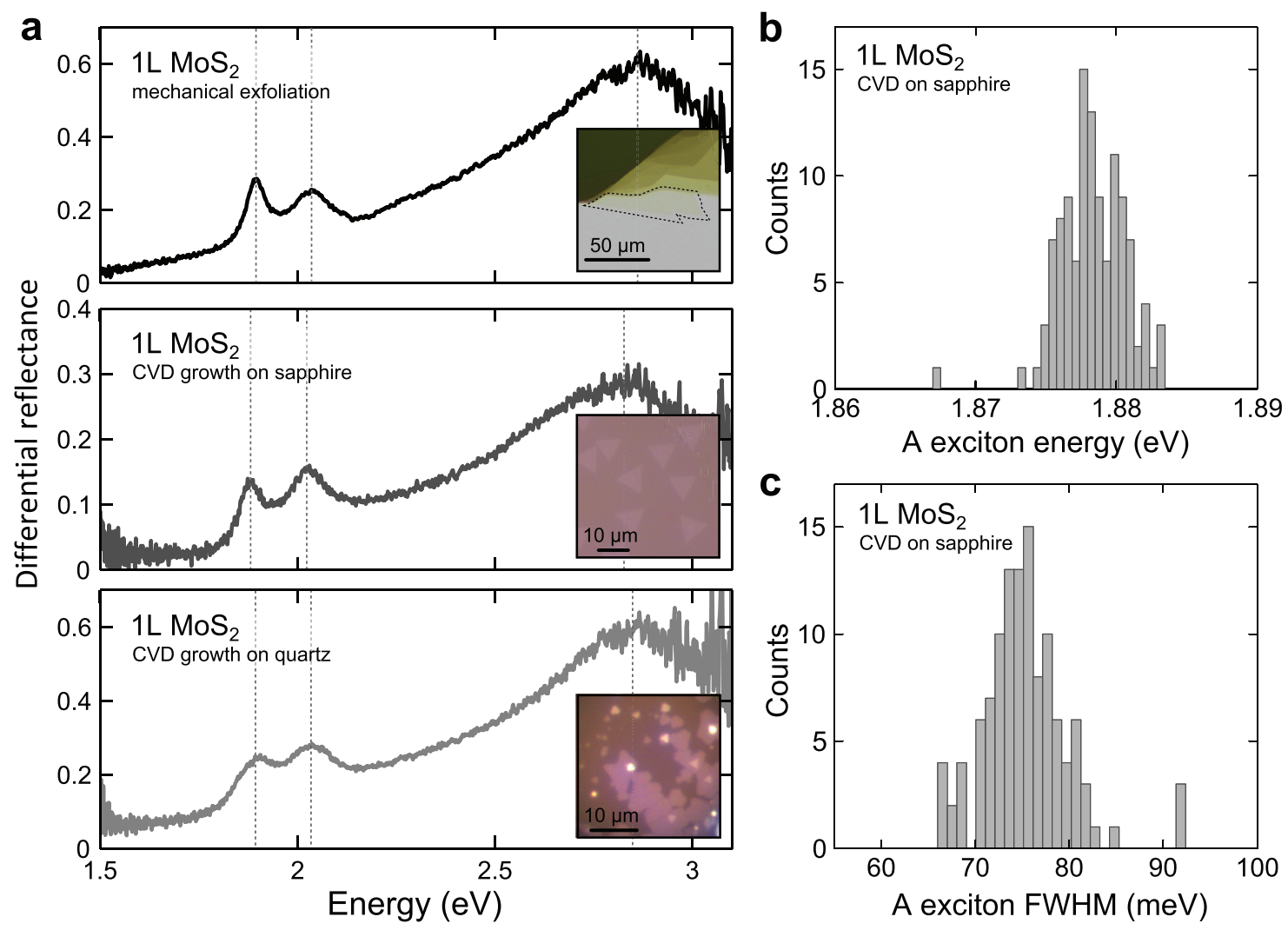

Figure 5. Differential reflectance spectra acquired on single-layer $\mathrm{MoS}_{2}$ samples prepared by mechanical exfoliation on PDMS (top panel in (a)), CVD growth on sapphire (middle panel in (a)) and CVD growth on quartz (bottom panel in (a)). The insets in (a) show optical images of the studied samples. (b) and (c) The statistical analysis of the energy and full-width at half maximum of the A exciton peak measured over $116 \mathrm{MoS}_{2}$ crystallites on the CVD sample grown on sapphire, showing the remarkable uniformity of the CVD grown material.

can thus use the position of the $\mathrm{C}$ exciton peak to independently determine the number of layers of $\mathrm{MoS}_{2}$ [14]. Note that photoluminescence measurements are typically limited to the study of the A and B excitons, as the excitation laser employed is typically in the $2.0-2.3 \mathrm{eV}$ range $[4,16]$. Figure $3(\mathrm{~b})$ summarizes the thickness dependence of the $\mathrm{A}, \mathrm{B}$ and $\mathrm{C}$ exciton resonances. A more comprehensive study of the thickness dependent excitonic features in W- and Mo-based TMDCs will be published elsewhere.

The micro-reflectance measurements of $\mathrm{MoS}_{2}$ flakes with different thicknesses (figures 3(a)-(c)) are compared with results obtained with Raman spectroscopy. Raman spectroscopy is a well-established technique to determine the number of layers of $\mathrm{MoS}_{2}$, as the frequency difference between the two most prominent Raman modes (the $A_{1 \mathrm{~g}}$ and the $E_{2 \mathrm{~g}}^{1}$ peaks) increases monotonically with the number of layers [9, 26]. Figure 3(d) shows the Raman spectra acquired on the $\mathrm{MoS}_{2}$ flakes on PDMS, with different numbers of layers. The thickness dependence of the two modes is displayed in figure 3(e). Figure 3(f) depicts the frequency difference between these two Raman modes. A direct comparison between figure 3(f) and figures 3(b), (c) indicates that micro-reflectance measurements are very effective for determining the number of layers, especially for multilayer flakes (thicker than four layers) where the frequency difference between the Raman modes starts to saturate.
It is important to note that micro-reflectance (or transmittance) measurements are very powerful for investigating indirect band gap semiconductors that typically require long exposure times in order to be studied by photoluminescence. In fact, the quantum yield of $\mathrm{MoS}_{2}$ strongly decreases by more than a factor of 1000 from the single layer case (direct band gap) to the multilayer case (indirect band gap) [5]. Moreover, these techniques can be used to study the optical properties of conducting and insulating materials without strong excitonic features, which constitutes another important advantage with respect to photoluminescence.

In order to illustrate the flexibility and versatility of this technique for identifying and studying other 2D materials we acquired differential reflectance spectra for single-layer flakes of other TMDCs. Figure 4 shows a comparison between the differential reflectance spectra of single-layer $\mathrm{WS}_{2}, \mathrm{MoS}_{2}$, $\mathrm{WSe}_{2}$ and $\mathrm{MoSe}_{2}$. The excitonic resonances are labelled according to the standard notation employed in the literature. One can easily identify the material from the position of the A and B exciton peaks (see table 2), which are in very good agreement with the reported values from photoluminescence experiments $[4,17,26,27]$. The origin of these A and $\mathrm{B}$ excitonic resonance peaks in $\mathrm{WS}_{2}, \mathrm{WSe}_{2}$ and $\mathrm{MoSe}_{2}$ is very similar to that in $\mathrm{MoS}_{2}$, i.e. the direct band gap transitions at the $\mathrm{K}$ point of the Brillouin zone (as sketched in the inset of figure 2(f)). Therefore, one can estimate the magnitude of the 
spin-orbit interaction in these materials from the energy difference between the A and B exciton. For W-based TMDCs this value is larger due to the higher atomic number of $\mathrm{W}$ with respect to Mo. Similarly, for the same transition metal, the Se-based TMDCs show greater spin-orbit interaction than the S-based ones. The C peak observed in $\mathrm{WS}_{2}$ and $\mathrm{MoSe}_{2}$ is believed to have the same origin as the one observed in $\mathrm{MoS}_{2}$ $[14,15,20-24,28]$. In the case of $\mathrm{WSe}_{2}$ several transitions along the $\bar{K} \bar{\Gamma}$ direction contribute to the $\mathrm{C}$ exciton. The $\mathrm{D}$ exciton transitions stem from the same bands as in the case of the B exciton, but occur along the $\overline{K M}$ direction $[17,27]$.

Finally, we demonstrate the suitability of differential reflectance for characterizing 2D materials prepared using large area growth methods such as chemical vapor deposition (CVD). Figure 5(a) compares the differential reflectance spectra acquired for monolayers of $\mathrm{MoS}_{2}$ prepared by mechanical exfoliation and by CVD growth. Two different sets of CVDprepared samples are characterized (see the supplementary material, available at stacks.iop.org/JPhysD/50/074002/ mmedia, for details on the growth methods). One has been grown on a highly polished sapphire substrate and results in characteristic single-crystal domains in the shape of welldefined equilateral triangles [29]. The other CVD sample has been grown directly on a quartz substrate. The energy of the excitonic peaks of the CVD samples is consistent with that measured on mechanically exfoliated single-layer $\mathrm{MoS}_{2}$, although it is slightly red-shifted. This red-shift in CVD-grown samples is typically attributed to strain induced during the thermal cycling during growth [30]. Differential reflectance spectroscopy is therefore also a powerful tool for studying CVD-grown samples. Moreover, the fast measurement time (approximately 1 second per spectrum) allows one to analyze the quality of a CVD-grown sample statistically by acquiring spectra over a large number of sample positions. Figures 5(b) and (c) show histograms of the energy and full width at half maximum of the A exciton peak measured on 116 different $\mathrm{MoS}_{2}$ triangular-shaped crystallites grown on the sapphire substrate, demonstrating the high uniformity of the CVD sample.

\section{Conclusions}

In summary, we have presented a simple and versatile optical microscope setup for carrying out micro-reflectance and transmittance spectroscopy with a lateral resolution of $\sim 1 \mu \mathrm{m}$ in the spectral range $400 \mathrm{~nm}-900 \mathrm{~nm}$. The setup can be used to unambiguously identify different $2 \mathrm{D}$ materials and determine their thicknesses, and also to characterize their fundamental optical properties. We believe that the presented setup can be replicated easily by other groups, as it is mainly based on standard commercially available optical components that can be purchased for a price of less than $9000 €$. Moreover, existing optical microscope setups can be upgraded easily by purchasing only some of the components.

\section{Acknowledgments}

We acknowledge funding from the European Commission under the Graphene Flagship, contract CNECTICT-604391.
AC-G acknowledges financial support from the MINECO (Ramón y Cajal 2014 program RYC-2014-01406 and MAT2014-58399-JIN) and from the Comunidad de Madrid (MAD2D-CM Program (S2013/MIT-3007)). RF acknowledges support from the Netherlands Organisation for Scientific Research (NWO) through the research program Rubicon with project number 680-50-1515. DPdL acknowledges support from the MINECO (program FIS2015-67367-C2-1-P). YN acknowledges a grant from the China Scholarship Council (file no. 201506120102).

\section{References}

[1] Novoselov K S, Geim A K, Morozov S V, Jiang D, Zhang Y, Dubonos S V, Grigorieva I V and Firsov A A 2004 Electric field effect in atomically thin carbon films Science 306 666-9

[2] Ferrari A C et al 2006 Raman spectrum of graphene and graphene layers Phys. Rev. Lett. 97187401

[3] Blake P, Hill E W, Castro Neto A H, Novoselov K S, Jiang D, Yang R, Booth T J and Geim A K 2007 Making graphene visible Appl. Phys. Lett. 91063124

[4] Splendiani A, Sun L, Zhang Y, Li T, Kim J, Chim C-Y, Galli G and Wang F 2010 Emerging photoluminescence in monolayer $\mathrm{MoS}_{2}$ Nano Lett. 10 1271-5

[5] Mak K F, Lee C, Hone J, Shan J and Heinz T F 2010 Atomically thin $\mathrm{MoS}_{2}$ : a new direct-gap semiconductor Phys. Rev. Lett. 105136805

[6] Abergel D S L, Russell A and Fal'ko V I 2007 Visibility of graphene flakes on a dielectric substrate Appl. Phys. Lett. 91063125

[7] Castellanos-Gomez A, Agraï N and Rubio-Bollinger G 2010 Optical identification of atomically thin dichalcogenide crystals Appl. Phys. Lett. 96213116

[8] Li H, Lu G, Yin Z, He Q, Li H, Zhang Q and Zhang H 2012 Optical identification of single- and few-layer $\mathrm{MoS}_{2}$ sheets Small 8 682-6

[9] Lee C, Yan H, Brus L E, Heinz T F, Hone Ḱ J and Ryu S 2010 Anomalous lattice vibrations of single-and few-layer $\mathrm{MoS}_{2}$ ACS Nano 4 2695-700

[10] Korn T, Heydrich S, Hirmer M, Schmutzler J and Schüller C 2011 Low-temperature photocarrier dynamics in monolayer $\mathrm{MoS}_{2}$ Appl. Phys. Lett. 99102109

[11] Zeng H, Dai J, Yao W, Xiao D and Cui X 2012 Valley polarization in $\mathrm{MoS}_{2}$ monolayers by optical pumping Nat. Nanotechnol. 7 490-3

[12] Mak K F, He K, Shan J and Heinz T F 2012 Control of valley polarization in monolayer $\mathrm{MoS}_{2}$ by optical helicity Nat. Nanotechnol. 7 494-8

[13] Jones A M et al 2013 Optical generation of excitonic valley coherence in monolayer $\mathrm{WSe}_{2}$ Nat. Nanotechnol. 8 634-8

[14] Dhakal K P, Duong D L, Lee J, Nam H, Kim M, Kan M, Lee Y H and Kim J 2014 Confocal absorption spectral imaging of $\mathrm{MoS}_{2}$ : optical transitions depending on the atomic thickness of intrinsic and chemically doped $\mathrm{MoS}_{2}$ Nanoscale 6 13028-35

[15] Kozawa D et al 2014 Photocarrier relaxation pathway in two-dimensional semiconducting transition metal dichalcogenides Nat. Commun. 5 193-335

[16] Mak K F, He K, Lee C, Lee G H, Hone J, Heinz T F and Shan J 2013 Tightly bound trions in monolayer $\mathrm{MoS}_{2}$ Nat. Mater. 12 207-11

[17] Zhao W, Ghorannevis Z, Chu L, Toh M, Kloc C, Tan P-H and Eda G 2013 Evolution of electronic structure in atomically thin sheets of $\mathrm{WS}_{2}$ and $\mathrm{WSe}_{2}$ ACS Nano 7 791-7 
[18] McIntyre J D E and Aspnes D E 1971 Differential reflection spectroscopy of very thin surface films Surf. Sci. 24 417-34

[19] Buscema M, Steele G A, van der Zant H S J and CastellanosGomez A 2014 The effect of the substrate on the Raman and photoluminescence emission of single-layer $\mathrm{MoS}_{2}$ Nano Res. 7 561-71

[20] Qiu D Y, da Jornada F H and Louie S G 2013 Optical spectrum of $\mathrm{MoS}_{2}$ : many-body effects and diversity of exciton states Phys. Rev. Lett. 111216805

[21] Klots A R et al 2014 Probing excitonic states in suspended two-dimensional semiconductors by photocurrent spectroscopy Sci. Rep. 46608

[22] Castellanos-Gomez A, Quereda J, van der Meulen H P, Agraït N and Rubio-Bollinger G 2016 Spatially resolved optical absorption spectroscopy of single- and few-layer $\mathrm{MoS}_{2}$ by hyperspectral imaging Nanotechnology 27115705

[23] Yu Y et al 2015 Exciton-dominated dielectric function of atomically thin $\mathrm{MoS}_{2}$ films Sci. Rep. 516996

[24] Gillen R and Maultzsch J 2017 Light-matter interactions in two-dimensional transition metal dichalcogenides: dominant excitonic transitions in mono- and few-layer
$\mathrm{MoX}_{2}$ and band nesting IEEE J. Sel. Top. Quantum Electron. 23 1-12

[25] Komsa H-P and Krasheninnikov A V 2012 Effects of confinement and environment on the electronic structure and exciton binding energy of $\mathrm{MoS}_{2}$ from first principles Phys. Rev. B 86241201

[26] Tonndorf P et al 2013 Photoluminescence emission and Raman response of monolayer $\mathrm{MoS}_{2}, \mathrm{MoSe}_{2}$, and $\mathrm{WSe}_{2}$ Opt. Express 21 4908-16

[27] Schmidt R, Niehues I, Schneider R, Drüppel M, Deilmann T, Rohlfing M, de Vasconcellos S M, Castellanos-Gomez A and Bratschitsch R 2016 Reversible uniaxial strain tuning in atomically thin $\mathrm{WSe}_{2} 2$ D Mater. 3021011

[28] Chernikov A, Berkelbach T C, Hill H M, Rigosi A, Li Y, Aslan O B, Reichman D R, Hybertsen M S and Heinz T F 2014 Exciton binding energy and nonhydrogenic Rydberg series in monolayer $\mathrm{WS}_{2}$ Phys. Rev. Lett. 113076802

[29] Dumcenco D et al 2015 Large-area epitaxial monolayer $\mathrm{MoS}_{2}$ ACS Nano 9 4611-20

[30] Liu Z et al 2014 Strain and structure heterogeneity in $\mathrm{MoS}_{2}$ atomic layers grown by chemical vapour deposition Nat. Commun. 55246 